\title{
Gender Effects on Statin-Associated Muscular Adverse Events: An Analysis of the FDA AERS Database
}

\author{
Hiroyuki Murakami ${ }^{1}$, Toshiyuki Sakaeda ${ }^{{ }^{*}}$, Kaori Kadoyama ${ }^{1}$, Yasushi Okuno ${ }^{2 *}$ \\ ${ }^{1}$ Center for Integrative Education in Pharmacy and Pharmaceutical Sciences, Graduate School of Pharmaceutical Sciences, Kyoto \\ University, Kyoto, Japan; ${ }^{2}$ Department of Systems Biosciences for Drug Discovery, Graduate School of Pharmaceutical Sciences, \\ Kyoto University, Kyoto, Japan. \\ Email: *sakaedat@pharm.kyoto-u.ac.jp, "okuno@pharm.kyoto-u.ac.jp
}

Received March $18^{\text {th }}, 2013$; revised May $2^{\text {nd }}, 2013$; accepted May $17^{\text {th }}, 2013$

Copyright (c) 2013 Hiroyuki Murakami et al. This is an open access article distributed under the Creative Commons Attribution License, which permits unrestricted use, distribution, and reproduction in any medium, provided the original work is properly cited.

\begin{abstract}
Objective: The reports submitted to the US Food and Drug Administration (FDA) Adverse Event Reporting System (FAERS) from 1997 to 2011 were reviewed to assess the gender effects on muscular adverse events induced by the administration of 3-hydroxy-3-methylglutaryl coenzyme A (HMG-CoA) reductase inhibitors (statins). Methods: After the deletion of duplicated submissions and the revision of arbitrary drug names, the reports involving pravastatin, simvastatin, atorvastatin, rosuvastatin, and cerivastatin were analyzed. Data mining algorithms were applied for the quantitative detection of signals, where a signal means a drug-associated adverse event, including the proportional reporting ratio, the reporting odds ratio, the information component, and the empirical Bayes geometric mean. Myopathy, myalgia, myositis, rhabdomyolysis, and an increase in creatine phosphokinase level were focused on as the muscular adverse events. Results: The total number of reports was 3,472,494. The signal scores suggested that all 5 statins were associated with 5 muscular adverse events in both male and female patients. The scores varied among statins, but were more noteworthy for cerivastatin. Conclusion: The data strongly suggested the necessity of well-organized clinical studies on statin-associated muscular adverse events.
\end{abstract}

Keywords: Adverse Event; FAERS; Statin; Data Mining; Pharmacovigilance

\section{Introduction}

Cardiovascular disease (CVD) is one of leading causes of death worldwide, and a high level of LDL-cholesterol in blood is an important risk factor for CVD [1-3]. The 3-hydroxy-3-methylglutaryl coenzyme A (HMG-CoA) reductase inhibitors, known as statins, are well-established therapeutic intervention for lowering of LDLcholesterol, and therefore the primary and secondary prevention of CVD [1-3]. Adverse events most commonly found in statin users are muscle symptoms, ranging from mild myalgia to life-threatening rhabdomyolysis requiring hospitalization [4-6]. Statin-associated muscular symptoms may affect $10 \%$ to $15 \%$ of users, resulting in autonomously discontinuation [4]. A better understanding of this important issue would therefore improve the management of patients [4-6].

In 2002, a guideline for managing statin-related myopathy was issued by clinical advisory of the American

${ }^{*}$ Corresponding authors.
College of Cardiology (ACC), the American Heart Association (AHA), and the National Heart, Lung, and Blood Institute (NHLBI) [7], based on information compiled by the US Food and Drug Administration (FDA), findings from clinical trials, and reports of the Adult Treatment Panel III (ATPIII) of the National Cholesterol Education Program (NCEP) [7]. The risk factors for myopathy included advanced age, female sex, small body frame and frailty, chronic renal insufficiency, multiple medications, and perioperative period [7-10], and various types of studies have continuously performed to support these information.

In 2005, the results of the PRIMO study, an observational study concerning the statin-associated muscular symptoms in unselected 7924 patients was published: the symptoms were reported by 832 patients (10.5\%), with a median time of 1 month following the initiation of statin therapy, but advanced age and female sex were not found to be risk factors [11]. In 2006, however, Jacobson published a review of the New Drug Applications (NDAs) 
and the FDA web site, in which a higher incidence of myopathy was found in thin, elderly women [12]. Later, a nested case-controlled study was conducted within a cohort of more than 250,000 new users of lipid-lowering medications, and it was suggested that advanced age was a risk factor for rhabdomyolysis, whereas female sex was not [13]. Recently, Hippisley-Cox and Coupland published the results of population-based cohort studies, in which more than 500,000 new statin users were compared with more than 3,500,000 non-users, and suggested that moderate to severe myopathy was more frequently occurred with the use of statins, but was more predominant for men than women $[14,15]$. In the results of a genome-wide study on statin-induced myopathy performed by the SEARCH Collaborative Group, advanced age was a risk factor, but the association with female sex depended on duration of therapy [16]. An assessment of statin safety by The National Lipid Association's (NLA) Muscle Safety Expert Panel listed elderly patients, not female patients, as the risk factors of myopathy and rhabdomyolysis [17]. Various factors contribute to these discrepancies, but female sex might have little effect on statin-associated muscular symptoms.

In this study, a total of 4,671,217 reports submitted to the FDA Adverse Event Reporting System (FAERS) from 1997 to 2011 were analyzed to assess gender effects on statin-associated muscular adverse events. The statins analyzed were pravastatin, fluvastatin, lovastatin, simvastatin, atorvastatin, rosuvastatin, pitavastatin, and cerivastatin. The muscular adverse events analyzed included myopathy, myalgia, myositis, rhabdomyolysis, and an increase in creatine phosphokinase level (CPK). Data mining algorithms were used for the quantitative detection of signals [18-26], where a signal means a statistical association between a drug and an adverse event or a drug-associated adverse event.

\section{Methods}

\subsection{Data Sources}

Input data for this study were taken from the public release of the FDA's AERS database, which covers the period from the 4th quarter of 1997 through the 3rd quarter of 2011. The total number of reports was 4,671,217. Besides those from manufacturers, reports can be submitted from health care professionals and the public. The informatics structure of the FAERS database adheres to the international safety reporting guidance issued by the International Conference on Harmonisation, ICH E2B. A data set consists of 7 data tables; patient demographic and administrative information (DEMO), drug/biologic information (DRUG), adverse events (REAC), patient outcomes (OUTC), report sources (RPSR), drug therapy start and end dates (THER), and indications for use/diagnosis (INDI). The adverse events in the REAC table are coded using the preferred terms (PTs) in the Medical Dictionary for Regulatory Activities (MedDRA) terminology, with version 15.0 used in this study.

Prior to data mining, duplicated reports were deleted according to the FDA's recommendation of adopting the most recent CASE number, resulting in a reduction in the number of reports from 4,671,217 to 3,472,494. All drug names were unified into generic names by a text-mining approach, because the FAERS system permits the registering of arbitrary drug names, including trade names and abbreviations. Spelling errors were detected by a spell checker software, GNU Aspell, and carefully confirmed by working pharmacists. Foods, beverages, treatments (e.g. X-ray radiation), and unspecified names (e.g. beta-blockers) were omitted. A total of 47,414,272 cooccurrences were found in 3,472,494 reports, where a cooccurrence was a pair of a drug and an adverse drug event.

\subsection{Definition of Adverse Events}

Myopathy, myalgia, myositis, rhabdomyolysis, and an increase in CPK are coded to PT10028641, PT10028411, PT10028653, PT10039020, and PT10005470, with 13, 18, 7, 2, and 12 lower level of terms (LLTs) assigned, respectively.

\subsection{Data Mining}

Data mining algorithms have been developed to identify drug-associated adverse events (signals) that are reported more frequently than expected by estimating expected reporting frequencies on the basis of information on all drugs and all events in the database [22-26]. For example, the proportional reporting ratio (PRR) [18], the reporting odds ratio (ROR) [19], the information component (IC) [20], and the empirical Bayes geometric mean (EBGM) [21] are widely used, and indeed, currently employed by the Medicines and Healthcare products Regulatory Agency (MHRA), UK, the Netherlands Pharmacovigilance Centre, the World Health Organization (WHO), and the FDA, respectively.

All of these algorithms calculate signal scores, i.e., the values for PRR, ROR, IC, and EBGM, to assess whether a drug is significantly associated with an adverse event or not from a two-by-two frequency table of counts. These calculations or algorithms, so-called the disproportionality analyses or measures, however, differ from one another in that the PRR and ROR are frequentist (nonBayesian), whereas the IC and EBGM are Bayesian.

In this section, only the scoring thresholds are given. The reader is referred to the articles for more extensive details on each statistical test [18-21]. Using the PRR, a 
signal is detected if the number of co-occurrences is 3 or more and the PRR is 2 or more with an associated $\chi^{2}$ value of 4 or more [18]. For the ROR, a signal is detected, if the lower limit of the $95 \%$ two-sided confidence interval exceeds 1 [19]. Signal detection using the IC is done using the IC025 metric, a lower limit of the 95\% twosided confidence interval of the IC, and a signal is detected if the IC025 value exceeds 0 [20]. Finally, for the EBGM, the EB05 metric, a lower one-sided 95\% confidence limit of the EBGM, is used and a signal is detected when the EB05 is greater than or equal to the threshold value 2.0 [21]. In this study, the adverse events were listed as drug-associated, when at least 1 of 4 indices met the criteria indicated above.

\section{Results}

The total number of statin-associated adverse events was 764 for pravastatin, 687 for fluvastatin, 599 for lovastatin, 885 for simvastatin, 1043 for atorvastatin, 850 for rosuvastatin, 320 for pitavastatin, and 205 for cerivastatin. The total number of co-occurrences with pravastatin was 123,224 , and 33,489 for fluvastatin, 45,731 for lovastatin, 390,420 for simvastatin, 438,317 for atorvastatin, 115,282 for rosuvastatin, 2559 for pitavastatin, and 66,622 for cerivastatin. The values for fluvastatin, lovas- tatin, and pitavastatin were not large enough to compare with the other 5 statins.

Gender data were available for 3,190,440 of 3,472,494 (91.9\%) reports; 1,260,182 reports for males and 1,930,258 reports for females. The statistical data on statin-associated muscular adverse events in all patients are listed in Table 1. The signal scores suggested that all 5 statins were associated with 5 muscular adverse events. The data on male and female patients are listed in Tables $\mathbf{2}$ and 3, respectively. Based on the PRR, ROR, or IC metrics, all associations were detected, but the EBGM failed to detect 5 signals. The signal scores varied among statins, but were more noteworthy for cerivastatin.

\section{Discussion}

Previously, we reviewed the FAERS database from 2004 to 2009 in order to assess the muscular and renal adverse events induced by statins [27]. Based on 1,644,220 reports, the signals were detected for pravastatin, simvastatin, atorvastatin, and rosuvastatin with respect to myalgia, rhabdomyolysis, and an increase in CPK, and these signals were stronger for rosuvastatin than pravastatin and atorvastatin [27]. These results were re-observed in this analysis (Table 1), and also for males (Table 2) and females (Table 3). However in this analysis, the stronger

Table 1. Signal scores for statin-associated muscular adverse events in all patients.

\begin{tabular}{|c|c|c|c|c|c|c|}
\hline & Statins & $\mathrm{N}$ & PRR (kai2) & ROR (95\% CI) & IC (95\% CI) & EBGM (95\% CI) \\
\hline \multirow[t]{5}{*}{ Myopathy } & Pravastatin & 129 & $3.02(172.9)^{*}$ & $3.04(2.56,3.52)^{*}$ & $1.57(1.32,1.82)^{*}$ & $2.92(2.52)^{*}$ \\
\hline & Simvastatin & 644 & $4.77(1925.1)^{*}$ & $4.92(4.55,5.29)^{*}$ & $2.24(2.13,2.35)^{*}$ & $4.74(4.44)^{*}$ \\
\hline & Atorvastatin & 594 & $3.91(1296.0)^{*}$ & $4.02(3.71,4.34)^{*}$ & $1.96(1.84,2.08)^{*}$ & $3.88(3.63)^{*}$ \\
\hline & Rosuvastatin & 142 & $3.56(258.8)^{*}$ & $3.58(3.03,4.12)^{*}$ & $1.80(1.56,2.04)^{*}$ & $3.44(2.98)^{*}$ \\
\hline & Cerivastatin & 499 & $21.77(9805.6)^{*}$ & $22.42(20.50,24.33)^{*}$ & $4.37(4.25,4.50)^{*}$ & $21.56(20.02)^{*}$ \\
\hline \multirow[t]{5}{*}{ Myalgia } & Pravastatin & 1427 & $3.42(2412.7)^{*}$ & $3.44(3.26,3.61)^{*}$ & $1.76(1.68,1.83)^{*}$ & $3.38(3.23)^{*}$ \\
\hline & Simvastatin & 4607 & $3.48(8112.5)^{*}$ & $3.55(3.45,3.66)^{*}$ & $1.79(1.74,1.83)^{*}$ & $3.45(3.36)^{*}$ \\
\hline & Atorvastatin & 6500 & $4.39(16900.7)^{*}$ & $4.53(4.42,4.64)^{*}$ & $2.12(2.08,2.15)^{*}$ & $4.34(4.25)^{*}$ \\
\hline & Rosuvastatin & 2750 & $7.12(14157.1)^{*}$ & $7.23(6.96,7.50)^{*}$ & $2.80(2.74,2.85)^{*}$ & $6.98(6.76)^{*}$ \\
\hline & Cerivastatin & 2299 & $10.42(18915.0)^{*}$ & $10.55(10.12,10.99)^{*}$ & $3.33(3.27,3.39)^{*}$ & $10.09(9.75)^{*}$ \\
\hline \multirow[t]{4}{*}{ Myositis } & Pravastatin & 115 & $3.28(180.3)^{*}$ & $3.30(2.75,3.85)^{*}$ & $1.68(1.41,1.94)^{*}$ & $3.15(2.69)^{*}$ \\
\hline & Simvastatin & 602 & $5.42(2181.5)^{*}$ & $5.63(5.19,6.07)^{*}$ & $2.43(2.31,2.54)^{*}$ & $5.40(5.05)^{*}$ \\
\hline & Atorvastatin & 407 & $3.26(642.0)^{*}$ & $3.33(3.02,3.65)^{*}$ & $1.70(1.55,1.84)^{*}$ & $3.22(2.97)^{*}$ \\
\hline & Cerivastatin & 254 & $13.44(2904.6)^{*}$ & $13.68(12.08,15.28)^{*}$ & $3.67(3.49,3.85)^{*}$ & $13.35(12.03)^{*}$ \\
\hline \multirow[t]{5}{*}{ Rhabdomyolysis } & Pravastatin & 577 & $2.49(510.4)^{*}$ & $2.50(2.30,2.69)^{*}$ & $1.31(1.19,1.42)^{*}$ & $2.46(2.30)^{*}$ \\
\hline & Simvastatin & 4232 & $5.79(16732.4)^{*}$ & $6.03(5.85,6.21)^{*}$ & $2.52(2.47,2.56)^{*}$ & $5.74(5.60)^{*}$ \\
\hline & Atorvastatin & 2086 & $2.53(1933.3)^{*}$ & $2.56(2.46,2.67)^{*}$ & $1.33(1.27,1.40)^{*}$ & $2.52(2.43)^{*}$ \\
\hline & Rosuvastatin & 888 & $4.10(2070.1)^{*}$ & $4.13(3.87,4.40)^{*}$ & $2.02(1.93,2.12)^{*}$ & $4.06(3.84)^{*}$ \\
\hline & Cerivastatin & 5576 & $48.28(236872.6)^{*}$ & $51.42(49.99,52.86)^{*}$ & $5.46(5.42,5.50)^{*}$ & $44.31(43.34)^{*}$ \\
\hline \multirow[t]{5}{*}{ An increase in CPK } & Pravastatin & 651 & $3.20(981.2)^{*}$ & $3.22(2.98,3.46)^{*}$ & $1.67(1.56,1.78)^{*}$ & $3.17(2.97)^{*}$ \\
\hline & Simvastatin & 2165 & $3.36(3600.4)^{*}$ & $3.43(3.29,3.57)^{*}$ & $1.74(1.68,1.80)^{*}$ & $3.34(3.23)^{*}$ \\
\hline & Atorvastatin & 2132 & $2.95(2752.8)^{*}$ & $3.00(2.87,3.13)^{*}$ & $1.55(1.49,1.62)^{*}$ & $2.93(2.83)^{*}$ \\
\hline & Rosuvastatin & 708 & $3.73(1404.0)^{*}$ & $3.75(3.48,4.02)^{*}$ & $1.88(1.78,1.99)^{*}$ & $3.69(3.46)^{*}$ \\
\hline & Cerivastatin & 942 & $8.65(6282.2)^{*}$ & $8.74(8.19,9.29)^{*}$ & $3.08(2.99,3.18)^{*}$ & $8.53(8.09)^{*}$ \\
\hline
\end{tabular}

$\mathrm{N}$ : the number of co-occurrences; PRR: the proportional reporting ratio; ROR: the reporting odds ratio; IC: the information component; EBGM: the empirical Bayes geometric mean; CI: the confidence interval (two-sided for ROR and IC, and one-sided for EBGM). An asterisk $\left({ }^{*}\right)$ indicates a statistically significant association, i.e., the adverse events are detected as signals. 
Table 2. Signal scores for statin-associated muscular adverse events in male patients.

\begin{tabular}{|c|c|c|c|c|c|c|}
\hline & Statins & $\mathrm{N}$ & PRR (kai2) & ROR (95\% CI) & IC $(95 \%$ CI) & EBGM (95\% CI) \\
\hline \multirow[t]{5}{*}{ Myopathy } & Pravastatin & 56 & $2.23(37.0)^{*}$ & $2.24(1.72,2.76)^{*}$ & $1.12(0.74,1.49)^{*}$ & $2.10(1.68)$ \\
\hline & Simvastatin & 374 & $4.45(1004.9)^{*}$ & $4.61(4.16,5.07)^{*}$ & $2.14(1.99,2.29)^{*}$ & $4.41(4.04)^{*}$ \\
\hline & Atorvastatin & 306 & $3.49(547.3)^{*}$ & $3.59(3.20,3.98)^{*}$ & $1.79(1.62,1.95)^{*}$ & $3.44(3.12)^{*}$ \\
\hline & Rosuvastatin & 69 & $3.37(112.5)^{*}$ & $3.39(2.67,4.10)^{*}$ & $1.69(1.35,2.03)^{*}$ & $3.14(2.56)^{*}$ \\
\hline & Cerivastatin & 216 & $17.00(3217.5)^{*}$ & $17.43(15.22,19.64)^{*}$ & $3.97(3.78,4.17)^{*}$ & $16.80(15.00)^{*}$ \\
\hline \multirow[t]{5}{*}{ Myalgia } & Pravastatin & 675 & $3.50(1191.3)^{*}$ & $3.52(3.27,3.78)^{*}$ & $1.79(1.68,1.90)^{*}$ & $3.45(3.23)^{*}$ \\
\hline & Simvastatin & 2325 & $3.59(4334.3)^{*}$ & $3.69(3.54,3.84)^{*}$ & $1.83(1.77,1.89)^{*}$ & $3.55(3.43)^{*}$ \\
\hline & Atorvastatin & 3063 & $4.56(8460.8)^{*}$ & $4.73(4.56,4.90)^{*}$ & $2.17(2.12,2.22)^{*}$ & $4.50(4.37)^{*}$ \\
\hline & Rosuvastatin & 1177 & $7.56(6538.6)^{*}$ & $7.68(7.24,8.11)^{*}$ & $2.88(2.79,2.96)^{*}$ & $7.39(7.04)^{*}$ \\
\hline & Cerivastatin & 986 & $10.24(7949.1)^{*}$ & $10.38(9.74,11.03)^{*}$ & $3.30(3.21,3.39)^{*}$ & $9.92(9.41)^{*}$ \\
\hline \multirow[t]{5}{*}{ Myositis } & Pravastatin & 72 & $3.69(138.8)^{*}$ & $3.72(2.95,4.49)^{*}$ & $1.82(1.49,2.15)^{*}$ & $3.46(2.82)^{*}$ \\
\hline & Simvastatin & 359 & $5.48(1322.9)^{*}$ & $5.75(5.17,6.33)^{*}$ & $2.43(2.28,2.59)^{*}$ & $5.46(5.00)^{*}$ \\
\hline & Atorvastatin & 196 & $2.87(239.8)^{*}$ & $2.93(2.54,3.32)^{*}$ & $1.50(1.30,1.71)^{*}$ & $2.81(2.49)^{*}$ \\
\hline & Rosuvastatin & 57 & $3.57(103.1)^{*}$ & $3.59(2.77,4.42)^{*}$ & $1.76(1.39,2.13)^{*}$ & $3.29(2.61)^{*}$ \\
\hline & Cerivastatin & 89 & $8.96(620.2)^{*}$ & $9.07(7.35,10.78)^{*}$ & $3.03(2.73,3.33)^{*}$ & $8.86(7.42)^{*}$ \\
\hline \multirow[t]{5}{*}{ Rhabdomyolysis } & Pravastatin & 301 & $2.00(149.7)^{*}$ & $2.01(1.79,2.22)^{*}$ & $0.99(0.83,1.15)^{*}$ & $1.98(1.80)$ \\
\hline & Simvastatin & 2498 & $4.99(7939.4)^{*}$ & $5.20(4.99,5.41)^{*}$ & $2.30(2.24,2.36)^{*}$ & $4.93(4.77)^{*}$ \\
\hline & Atorvastatin & 1065 & $2.03(555.8)^{*}$ & $2.05(1.93,2.17)^{*}$ & $1.01(0.93,1.10)^{*}$ & $2.02(1.92)$ \\
\hline & Rosuvastatin & 500 & $4.09(1153.4)^{*}$ & $4.12(3.77,4.47)^{*}$ & $2.01(1.88,2.14)^{*}$ & $4.02(3.73)^{*}$ \\
\hline & Cerivastatin & 2382 & $33.59(69346.5)^{*}$ & $35.23(33.75,36.71)^{*}$ & $4.93(4.87,5.00)^{*}$ & $30.95(29.92)^{*}$ \\
\hline \multirow[t]{5}{*}{ An increase in CPK } & Pravastatin & 383 & $2.84(451.8)^{*}$ & $2.85(2.58,3.13)^{*}$ & $1.49(1.34,1.63)^{*}$ & $2.79(2.56)^{*}$ \\
\hline & Simvastatin & 1306 & $2.88(1607.7)^{*}$ & $2.94(2.78,3.10)^{*}$ & $1.52(1.44,1.60)^{*}$ & $2.86(2.73)^{*}$ \\
\hline & Atorvastatin & 1246 & $2.64(1271.5)^{*}$ & $2.69(2.54,2.83)^{*}$ & $1.39(1.31,1.47)^{*}$ & $2.62(2.50)^{*}$ \\
\hline & Rosuvastatin & 441 & $4.00(983.4)^{*}$ & $4.03(3.67,4.39)^{*}$ & $1.98(1.84,2.12)^{*}$ & $3.94(3.64)^{*}$ \\
\hline & Cerivastatin & 396 & $5.79(1544.5)^{*}$ & $5.83(5.28,6.38)^{*}$ & $2.50(2.35,2.64)^{*}$ & $5.71(5.25)^{*}$ \\
\hline
\end{tabular}

$\mathrm{N}$ : the number of co-occurrences; PRR: the proportional reporting ratio; ROR: the reporting odds ratio; IC: the information component; EBGM: the empirical Bayes geometric mean; CI: the confidence interval (two-sided for ROR and IC, and one-sided for EBGM). An asterisk $\left({ }^{*}\right)$ indicates a statistically significant association, i.e., the adverse events are detected as signals.

association with rosuvastatin than pravastatin and atorvastatin was not observed for myopathy or myositis (Tables 1-3). Consequently, it can be concluded that statinassociated muscular adverse events occur in both male and female patients, but the strength of the association depends on types of both statins and muscular adverse events.

Cerivastatin, approved in 1997 by the FDA, was withdrawn from the US market in 2001, and thereafter safety became a central issue in the use of statins [12]. Consistent with this, signal scores were higher for cerivastatin than the other 4 statins (Tables 1-3). An analysis after stratification according to gender suggested that cerivastatin-associated muscular adverse events were more noteworthy in females than males (Tables 2 and 3). This was also consistent with a medical review by the FDA [12]. Although extensively high signal scores might, in part, be explained by the notoriety effect [28], the FAERS database and data mining algorithms used in this study are useful for pharmacoepidemiological studies and/or pharmacovigilance analyses.

Muscular symptoms are the most commonly found adverse events in statin users, but their prevalence varies among reports [4-6]. The NLA Muscle Safety Expert Panel and other statin experts have emphasized the importance of standardizing terms related to myopathy to allow reliable comparisons among research studies and to improve care for statin users [6]; however to date, no consensus exists on the definition of statin-related myopathy $[29,30]$. For example, ACC/AHA/NHLBI defines myopathy as a general term referring to any disease of muscles, whereas NLA defines it as symptoms of myalgia (muscle pain or soreness), weakness, or cramps, plus creatine kinase level more than 10 times the upper limit of normal $[29,30]$. The difference in the definition must be responsible for the variation in the prevalence of muscular symptoms among reports and also for discrepancies in the list of risk factors. From this point of view, the coding of adverse events using the PT terms in the MedDRA terminology in the FAERS database has considerable advantages, although data mining does not provide sufficient evidence on causality.

In conclusion, reports in the FAERS database were reviewed to assess gender effects on statin-associated muscular adverse events. Based on 3,472,494 reports from 1997 to 2011, it was suggested that all 5 statins were 
Table 3. Signal scores for statin-associated muscular adverse events in female patients.

\begin{tabular}{|c|c|c|c|c|c|c|}
\hline & Statins & $\mathrm{N}$ & PRR (kai2) & ROR (95\% CI) & IC (95\% CI) & EBGM (95\% CI) \\
\hline \multirow[t]{5}{*}{ Myopathy } & Pravastatin & 64 & $3.61(118.3)^{*}$ & $3.63(2.84,4.42)^{*}$ & $1.78(1.43,2.14)^{*}$ & $3.35(2.69)^{*}$ \\
\hline & Simvastatin & 240 & $4.55(664.2)^{*}$ & $4.66(4.10,5.23)^{*}$ & $2.16(1.97,2.34)^{*}$ & $4.49(4.02)^{*}$ \\
\hline & Atorvastatin & 225 & $3.55(412.8)^{*}$ & $3.63(3.18,4.08)^{*}$ & $1.81(1.62,2.00)^{*}$ & $3.48(3.10)^{*}$ \\
\hline & Rosuvastatin & 65 & $3.56(117.4)^{*}$ & $3.58(2.81,4.36)^{*}$ & $1.77(1.41,2.12)^{*}$ & $3.31(2.67)^{*}$ \\
\hline & Cerivastatin & 274 & $28.27(7132.7)^{*}$ & $29.29(25.95,32.63)^{*}$ & $4.67(4.50,4.85)^{*}$ & $27.92(25.25)^{*}$ \\
\hline \multirow[t]{5}{*}{ Myalgia } & Pravastatin & 714 & $3.27(1112.0)^{*}$ & $3.29(3.05,3.52)^{*}$ & $1.69(1.59,1.80)^{*}$ & $3.22(3.03)^{*}$ \\
\hline & Simvastatin & 2148 & $3.30(3430.0)^{*}$ & $3.36(3.21,3.50)^{*}$ & $1.71(1.65,1.77)^{*}$ & $3.27(3.15)^{*}$ \\
\hline & Atorvastatin & 3094 & $3.97(6842.7)^{*}$ & $4.07(3.93,4.22)^{*}$ & $1.97(1.92,2.03)^{*}$ & $3.93(3.81)^{*}$ \\
\hline & Rosuvastatin & 1509 & $6.79(7292.6)^{*}$ & $6.89(6.54,7.23)^{*}$ & $2.73(2.66,2.80)^{*}$ & $6.66(6.38)^{*}$ \\
\hline & Cerivastatin & 1286 & $10.98(11238.3)^{*}$ & $11.12(10.51,11.72)^{*}$ & $3.40(3.32,3.48)^{*}$ & $10.60(10.13)^{*}$ \\
\hline \multirow[t]{4}{*}{ Myositis } & Pravastatin & 42 & $2.69(43.1)^{*}$ & $2.70(2.00,3.41)^{*}$ & $1.36(0.92,1.79)^{*}$ & $2.44(1.88)$ \\
\hline & Simvastatin & 223 & $4.80(671.7)^{*}$ & $4.94(4.32,5.55)^{*}$ & $2.24(2.04,2.43)^{*}$ & $4.76(4.24)^{*}$ \\
\hline & Rosuvastatin & 36 & $2.24(23.6)^{*}$ & $2.25(1.62,2.88)^{*}$ & $1.10(0.63,1.57)^{*}$ & $2.04(1.55)$ \\
\hline & Cerivastatin & 149 & $17.42(2282.4)^{*}$ & $17.79(15.12,20.46)^{*}$ & $3.96(3.73,4.20)^{*}$ & $17.23(15.03)^{*}$ \\
\hline \multirow[t]{5}{*}{ Rhabdomyolysis } & Pravastatin & 250 & $2.91(311.2)^{*}$ & $2.92(2.58,3.27)^{*}$ & $1.52(1.34,1.70)^{*}$ & $2.85(2.56)^{*}$ \\
\hline & Simvastatin & 1457 & $5.72(5663.6)^{*}$ & $5.91(5.61,6.22)^{*}$ & $2.50(2.43,2.58)^{*}$ & $5.68(5.44)^{*}$ \\
\hline & Atorvastatin & 766 & $2.49(687.7)^{*}$ & $2.53(2.35,2.70)^{*}$ & $1.31(1.21,1.42)^{*}$ & $2.48(2.33)^{*}$ \\
\hline & Rosuvastatin & 330 & $3.74(657.0)^{*}$ & $3.76(3.37,4.15)^{*}$ & $1.88(1.73,2.04)^{*}$ & $3.67(3.35)^{*}$ \\
\hline & Cerivastatin & 2791 & $63.94(159170.3)^{*}$ & $69.11(66.39,71.83)^{*}$ & $5.85(5.79,5.91)^{*}$ & $58.83(57.02)^{*}$ \\
\hline \multirow[t]{5}{*}{ An increase in CPK } & Pravastatin & 236 & $3.19(351.5)^{*}$ & $3.20(2.82,3.59)^{*}$ & $1.65(1.47,1.84)^{*}$ & $3.11(2.79)^{*}$ \\
\hline & Simvastatin & 747 & $3.39(1258.6)^{*}$ & $3.45(3.20,3.69)^{*}$ & $1.75(1.65,1.86)^{*}$ & $3.36(3.16)^{*}$ \\
\hline & Atorvastatin & 728 & $2.75(812.7)^{*}$ & $2.79(2.59,2.99)^{*}$ & $1.45(1.35,1.56)^{*}$ & $2.73(2.56)^{*}$ \\
\hline & Rosuvastatin & 237 & $3.11(336.4)^{*}$ & $3.12(2.75,3.50)^{*}$ & $1.62(1.43,1.80)^{*}$ & $3.04(2.73)^{*}$ \\
\hline & Cerivastatin & 505 & $12.52(5272.1)^{*}$ & $12.71(11.63,13.79)^{*}$ & $3.59(3.47,3.72)^{*}$ & $12.34(11.46)^{*}$ \\
\hline
\end{tabular}

$\mathrm{N}$ : the number of co-occurrences; PRR: the proportional reporting ratio; ROR: the reporting odds ratio; IC: the information component; EBGM: the empirical Bayes geometric mean; CI: the confidence interval (two-sided for ROR and IC, and one-sided for EBGM). An asterisk ( ${ }^{*}$ ) indicates a statistically significant association, i.e., the adverse events are detected as signals.

associated with 5 muscular adverse events in both male and female patients. The strength of the association varied among statins, but was more noteworthy for cerivastatin. The data strongly suggested the necessity of wellorganized clinical studies on statin-associated muscular adverse events.

\section{REFERENCES}

[1] F. Taylor, K. Ward, T. H. Moore, M. Burke, G. Davey Smith, et al., "Statins for the Primary Prevention of Cardiovascular Disease," Cochrane Database of Systematic Reviews, No. 1, 2011, Article ID: CD004816. doi:10.1002/14651858.CD004816.pub4

[2] P. P. Toth, "Drug Treatment of Hyperlipidaemia: A Guide to the Rational Use of Lipid-Lowering Drugs," Drugs, Vol. 70, No. 11, 2010, pp. 1363-1379. doi:10.2165/10898610-000000000-00000

[3] J. A. Lardizabal and P. C. Deedwania, "Benefits of Statin Therapy and Compliance in High Risk Cardiovascular Patients," Vascular Health and Risk Management, Vol. 6, 2010, pp. 843-853. doi:10.2147/VHRM.S9474

[4] C. R. Harper and T. A. Jacobson, "Evidence-Based Management of Statin Myopathy,” Current Atherosclerosis
Reports, Vol. 12, No. 5, 2010, pp. 322-330. doi:10.1007/s11883-010-0120-9

[5] T. A. Jacobson, “Toward 'Pain-Free' Statin Prescribing: Clinical Algorithm for Diagnosis and Management of Myalgia,” Mayo Clinic Proceedings, Vol. 83, No. 6, 2008, pp. 687-700. doi:10.4065/83.6.687

[6] S. L. Di Stasi, T. D. MacLeod, J. D. Winters and S. A. Binder-Macleod, "Effects of Statins on Skeletal Muscle: A Perspective for Physical Therapists," Physical Therapy, Vol. 90, No. 10, 2010, pp. 1530-1542. doi:10.2522/ptj.20090251

[7] R. C. Pasternak, S. C. Smith Jr., C. N. Bairey-Merz, S. M. Grundy, J. I. Cleeman, et al., "ACC/AHA/NHLBI Clinical Advisory on the Use and Safety of Statins," Circulation, Vol. 106, No. 8, 2002, pp. 1024-1028. doi:10.1161/01.CIR.0000032466.44170.44

[8] S. S. Tomlinson and K. K. Mangione, "Potential Adverse Effects of Statins on Muscle,” Physical Therapy, Vol. 85, No. 5, 2005, pp. 459-465.

[9] Y. S. Chatzizisis, K. C. Koskinas, G. Misirli, C. Vaklavas, A. Hatzitolios, et al., "Risk Factors and Drug Interactions Predisposing to Statin-Induced Myopathy,” Drug Safety, Vol. 33, No. 3, 2010, pp. 171-187. doi:10.2165/11319380-000000000-00000

[10] B. A. Golomb and M. A. Evans, "Statin Adverse Effects: 
A Review of the Literature and Evidence for a Mitochondrial Mechanism," American Journal of Cardiovascular Drugs, Vol. 8, No. 6, 2008, pp. 373-418. doi:10.2165/0129784-200808060-00004

[11] E. Bruckert, G. Hayem, S. Dejager, C. Yau and B. Bégaud, "Mild to Moderate Muscular Symptoms with High-Dosage Statin Therapy in Hyperlipidemic Patientsthe PRIMO Study," Cardiovascular Drugs and Therapy, Vol. 19, No. 6, 2005, pp. 403-414. doi:10.1007/s10557-005-5686-Z

[12] T. A. Jacobson, "Statin Safety: Lessons from New Drug Applications for Marketed Statins," American Journal of Cardiology, Vol. 97, No. 8A, 2006, pp. 44C-51C. doi:10.1016/j.amjcard.2005.12.009

[13] S. Schech, D. Graham, J. Staffa, S. E. Andrade, L. L. Grenade, et al., "Risk Factors for Statin-Associated Rhabdomyolysis," Pharmacoepidemiology and Drug Safety, Vol. 16, No. 3, 2007, pp. 352-358. doi:10.1002/pds.1287

[14] J. Hippisley-Cox and C. Coupland, "Individualising the Risks of Statins in Men and Women in England and Wales: Population-Based Cohort Study,” Heart, Vol. 96, No. 12, 2010, pp. 939-947. doi:10.1136/hrt.2010.199034

[15] J. Hippisley-Cox and C. Coupland, "Unintended Effects of Statins in Men and Women in England and Wales: Population Based Cohort Study Using the QResearch Database,” British Medical Journal, Vol. 340, 2010, p. c2197. doi:10.1136/bmj.c2197

[16] SEARCH Collaborative Group, E. Link, S. Parish, J. Armitage, L. Bowman, S. Heath, et al., "SLCO1B1 Variants and Statin-Induced Myopathy-A Genomewide Study," New England Journal of Medicine, Vol. 359, No. 8, 2008, pp. 789-799. doi:10.1056/NEJMoa0801936

[17] P. D. Thompson, P. M. Clarkson and R. S. Rosenson, "National Lipid Association Statin Safety Task Force Muscle Safety Expert Panel. An Assessment of Statin Safety by Muscle Experts," American Journal of Cardiology, Vol. 97, No. 8A, 2006, pp. 69C-76C. doi:10.1016/j.amjcard.2005.12.013

[18] S. J. Evans, P. C. Waller and S. Davis, "Use of Proportional Reporting Ratios (PRRs) for Signal Generation from Spontaneous Adverse Drug Reaction Reports," Pharmacoepidemiology and Drug Safety, Vol. 10, No. 6, 2001, pp. 483-486. doi:10.1002/pds.677

[19] E. P. van Puijenbroek, A. Bate, H. G. Leufkens, M. Lindquist, R. Orre, et al., "A Comparison of Measures of Disproportionality for Signal Detection in Spontaneous Reporting Systems for Adverse Drug Reactions," Pharmacoepidemiology and Drug Safety, Vol. 11, No. 1, 2002, pp. 3-10. doi:10.1002/pds.668

[20] A. Bate, M. Lindquist, I. R. Edwards, S. Olsson, R. Orre, et al., "A Bayesian Neural Network Method for Adverse Drug Reaction Signal Generation,” European Journal of
Clinical Pharmacology, Vol. 54, No. 4, 1998, pp. 315321. doi:10.1007/s002280050466

[21] A. Szarfman, S. G. Machado and R. T. O’Neill, "Use of Screening Algorithms and Computer Systems to Efficiently Signal Higher-than-Expected Combinations of Drugs and Events in the US FDA's Spontaneous Reports Database,” Drug Safety, Vol. 25, No. 6, 2002, pp. 381392. doi:10.2165/00002018-200225060-00001

[22] A. Bate and S. J. Evans, "Quantitative Signal Detection Using Spontaneous ADR Reporting,” Pharmacoepidemiology and Drug Safety, Vol. 18, No. 6, 2009, pp. 427436. doi:10.1002/pds. 1742

[23] M. Hauben and L. Reich, "Drug-Induced Pancreatitis: Lessons in Data Mining," British Journal of Clinical Pharmacology, Vol. 58, No. 5, 2004, pp. 560-562. doi:10.1111/j.1365-2125.2004.02203.x

[24] J. Almenoff, J. M. Tonning, A. L. Gould, A. Szarfman, M. Hauben, et al., "Perspectives on the Use of Data Mining in Pharmacovigilance,” Drug Safety, Vol. 28, No. 11, 2005, pp. 981-1007. doi:10.2165/00002018-200528110-00002

[25] J. S. Almenoff, E. N. Pattishall, T. G. Gibbs, W. DuMouchel, S. J. Evans, et al., "Novel Statistical Tools for Monitoring the Safety of Marketed Drugs," Clinical Pharmacology and Therapeutics, Vol. 82, No. 2, 2007, pp. 157-166. doi:10.1038/sj.clpt.6100258

[26] M. Hauben and A. Bate, "Decision Support Methods for the Detection of Adverse Events in Post-Marketing Data," Drug Discovery Today, Vol. 14, No. 7-8, 2009, pp. 343357. doi:10.1016/j.drudis.2008.12.012

[27] T. Sakaeda, K. Kadoyama and Y. Okuno, "Statin-Associated Muscular and Renal Adverse Events: Data Mining of the Public Version of the FDA Adverse Event Reporting System,” PLoS ONE, Vol. 6, No.12, 2011, p. e28124. doi:10.1371/journal.pone.0028124

[28] A. Pariente, F. Gregoire, A. Fourrier-Reglat, F. Haramburu and N. Moore, "Impact of Safety Alerts on Measures of Disproportionality in Spontaneous Reporting Databases: The Notoriety Bias,” Drug Safety, Vol. 30, No. 10, 2007, pp. 891-898. doi:10.2165/00002018-200730100-00007

[29] J. M. McKenney, M. H. Davidson, T. A. Jacobson and J. R. Guyton, "Final Conclusions and Recommendations of the National Lipid Association Statin Safety Assessment Task Force," American Journal of Cardiology, Vol. 97, No. 8A, 2006, pp. 89C-94C. doi:10.1016/j.amjcard.2006.02.030

[30] T. R. Joy and R. A. Hegele, "Narrative Review: StatinRelated Myopathy," Annals of Internal Medicine, Vol. 150, No. 12, 2009, pp. 858-868. doi:10.7326/0003-4819-150-12-200906160-00009 


\section{Abbreviations}

ACC: the American College of Cardiology; AHA: the American Heart Association; ATPIII: the Adult Treatment Panel III;

CI: confidence interval;

CPK: creatine phosphokinase;

CVD: cardiovascular disease;

EBGM: empirical Bayes geometric mean;

FAERS: the FDA Adverse Event Reporting System;

FDA: the US Food and Drug Administration;

IC: information component;

ICH: the International Conference on Harmonisation;

LLT: lower level of term;
MedDRA: the Medical Dictionary for Regulatory Activities;

MHRA: the Medicines and Healthcare products Regulatory Agency;

NCEP: the National Cholesterol Education Program;

NDAs: the New Drug Applications;

NHLBI: the National Heart, Lung, and Blood Institute;

NLA: the National Lipid Association;

PT: preferred term;

PRR: proportional reporting ratio;

ROR: reporting odds ratio;

WHO: the World Health Organization. 